\title{
Valve-in-Valve-in-Valve: Degenerated Transcatheter Heart Valve within Degenerated Surgical Bioprosthetic Aortic Valve Treated with Second Transcatheter Heart Valve
}

\author{
Muhammad Ajmal $1,2, *$, Sridhar Reddy ${ }^{1,3}$, Ranjith Shetty ${ }^{1,3}$, Toshinobu Kazui ${ }^{4}$ and \\ Kapildeo Lotun ${ }^{1,3,5}$ \\ 1 Sarvar Heart Center, Tucson, AZ 85724, USA; srireddymd86@gmail.com (S.R.); \\ ranjith.shetty@carondelet.org (R.S.); klotun@hotmail.com (K.L.) \\ 2 Department of Cardiovascular Fellowship, University of Arizona, Tucson, AZ 85724, USA \\ 3 Department of Interventional Cardiology, University of Arizona, Tucson, AZ 85724, USA \\ 4 Department of Cardiothoracic Surgery, University of Arizona, Tucson, AZ 85724, USA; \\ toshinobu.kazui@bannerhealth.com \\ 5 Cardiac catheterization laboratory, Banner University Medical Center, Tucson, AZ 85724, USA \\ * Correspondence: drajmal207@gmail.com
}

Received: 24 February 2020; Accepted: 24 March 2020; Published: 25 March 2020

\begin{abstract}
Currently, transcatheter aortic valve replacements within degenerated surgical bioprosthetic aortic valves (valve in valve) are increasing in frequency with studies supporting their safety and efficacy. We present the rare case of a patient requiring a second transcatheter bioprosthetic aortic valve placed within a previously placed degenerated transcatheter aortic valve, which was implanted in a degenerated surgical bioprosthetic aortic valve. The procedure was performed using a percutaneous cardiopulmonary bypass with TandemLife for hemodynamic support.
\end{abstract}

Keywords: prosthetic aortic valve stenosis; cardiomyopathy; cardiogenic shock

\section{Introduction}

Transcatheter aortic valve replacement (TAVR) is gaining an increasingly important role in the treatment of symptomatic patients with severe aortic stenosis (AS) [1-3]. Due to concerns around the longevity and durability of bioprosthetic valves, the use of transcatheter heart valves (THV) within degenerated surgical bioprosthetic valves has become an important field of study, especially in high-risk surgical patients. Among patients that are at high risk of complications of surgical aortic valve replacement (AVR), valve-in-valve (ViV) TAVR is an attractive and safe alternative [4]. We report the case of an aortic valve replacement in a high-risk patient who had previously undergone ViV TAVR.

\section{History}

A 68-year-old male with a history of heart failure and reduced ejection fraction (HFrEF), and with a left ventricular ejection fraction (LVEF) of 30\%, hypertension, hyperlipidemia, and cirrhosis secondary to nonalcoholic steatotic hepatitis (NASH) was referred for symptomatic severe bioprosthetic aortic valve stenosis. He had a surgical AVR with a $21 \mathrm{~mm}$ Carpentier-Edwards PERIMOUNT aortic heart valve 13 years ago, followed by TAVR with a $23 \mathrm{~mm}$ Edwards Sapien XT valve three years ago. The bioprosthesis was initially selected because of a concern for the compliance with anticoagulation, and the patient had progressively worsening fatigue and dyspnea on exertion, and the transthoracic echocardiogram (TTE) showed severe aortic stenosis with a peak velocity of $4.1 \mathrm{~m} / \mathrm{s}$, mean aortic valve 
gradient of $40 \mathrm{mmHg}$ and area of $0.78 \mathrm{~cm}^{2}$. The aortic valve velocity is shown in Figure 1. Informed consent was obtained from the patient.

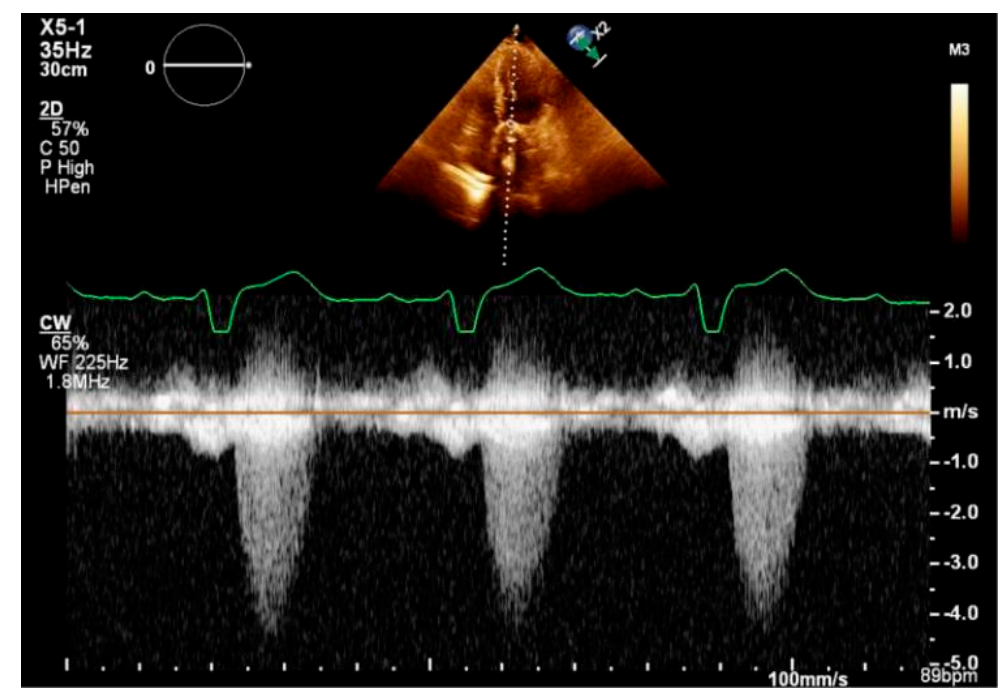

Figure 1. TTE showing peak aortic valve velocity.

He was deemed high risk for surgical AVR due to repeated sternotomy, HFrEF, cirrhosis, and frailty, and the Society of Thoracic Surgery (STS) risk score was calculated at $8.4 \%$. After a multi-disciplinary heart team discussion, it was decided to proceed with percutaneous approach to treat the prosthetic aortic valve stenosis. A plan was made to perform fracture of the Carpentier-Edwards PERIMOUNT valve in order to fully expand the existing $23 \mathrm{~mm}$ Edwards SAPIEN XT transcatheter heart valve (THV). The major risk for this procedure was acute valvular regurgitation, and the concern for the intolerance of this complication in the presence of low EF. As a result, we decided to have another THV on standby for deployment, choosing a $26 \mathrm{~mm}$ Medtronic CoreValve Evolut-R based on pre-TAVR planning computed tomography angiography. To avoid hemodynamic instability in the setting of prolonged rapid ventricular pacing during valve fracture and the potential risk of acute aortic regurgitation after valve fracture, a percutaneous cardiopulmonary bypass with the TandemLife ${ }^{\circledR}$ was undertaken. Furthermore, we were prepared to place an Impella ${ }^{\circledR} \mathrm{CP}$ percutaneous left ventricular device via the right axillary artery approach to decompress the left ventricle in case the patient required prolonged support with TandemLife in the setting of uncorrected acute severe aortic regurgitation.

\section{Procedure}

The procedure was performed under general anesthesia, using fluoroscopic and Transesophageal echocardiogram (TEE)guidance. Access for the TandemLife ${ }^{\circledR}$ was obtained in the left common femoral artery and right common femoral vein and both arteriotomy and veinotomy sites were "pre-closed" using two Perclose Proglide (Abbott Inc., Abbott Park, IL, USA) suture mediated vascular closure devices. A 22-French TandemLife ${ }^{\circledR}$ venous cannula in the right common femoral vein, and a 17-French TandemLife $^{\circledR}$ arterial cannulae in the left common femoral artery was placed. The cannulae were then connected to the TandemLife ${ }^{\circledR}$ pump attached to an oxygenator. A cardiopulmonary bypass was initiated at a flow rate of 1.5 to $2 \mathrm{~L}$ per minute. Next, a 5 French balloon-tipped transvenous pacer was placed via a 7 French sheath in the left common femoral vein. The transvenous pacer was placed at the apex of the right ventricle, and threshold testing was performed confirming excellent position of pacer and capture. The right common femoral artery was then accessed and "pre-closed" using two Perclose Proglide suture mediated closure devices. A 14 French dry Gore Sheath (Gore Inc., Newark, Delaware, USA) was placed in the right common femoral artery through which a 6 French Judkins Right 4 (JR4) catheter and stiff angled Glidewire (Terumo, Somerset, NJ, USA) was used to cross the aortic valve. The JR4 was exchanged for a 6 French pigtail catheter, through which an Amplatz Super Stiff wire 
(Boston Scientific, Boston, MA, USA) was advanced into the apex of the left ventricle. The $26 \mathrm{~mm}$ Medtronic CoreValve Evolut-R was prepped and loaded onto the back table, if needed. Over the Amplatz Super Stiff wire, a $22 \mathrm{~mm}$ True balloon (Bard Inc., Tempe, AZ, USA) was placed in the aortic valve position. Rapid right ventricular pacing was initiated at 180 beats per minute, and the True balloon was inflated up to 12 atmospheres as shown in Figure 2, at which time the Carpentier-Edwards Perimount surgical bioprosthetic valve was fractured.

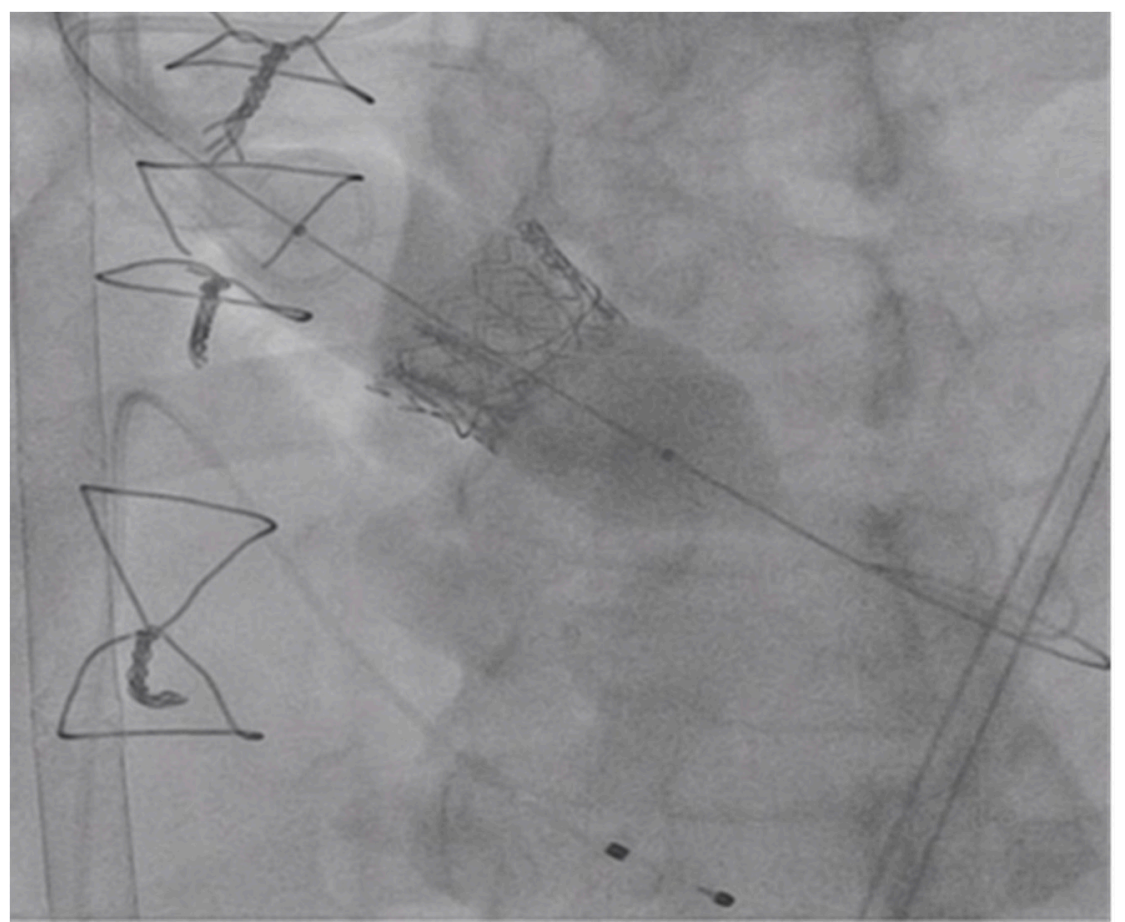

Figure 2. Left Anterior Oblique (LAO) Caudal View: True balloon fracturing the surgical bioprosthetic valve. The transvenous pacemaker in place.

Post valvuloplasty, the patient developed significant acute aortic regurgitation with hemodynamic instability as shown in Figure 3.

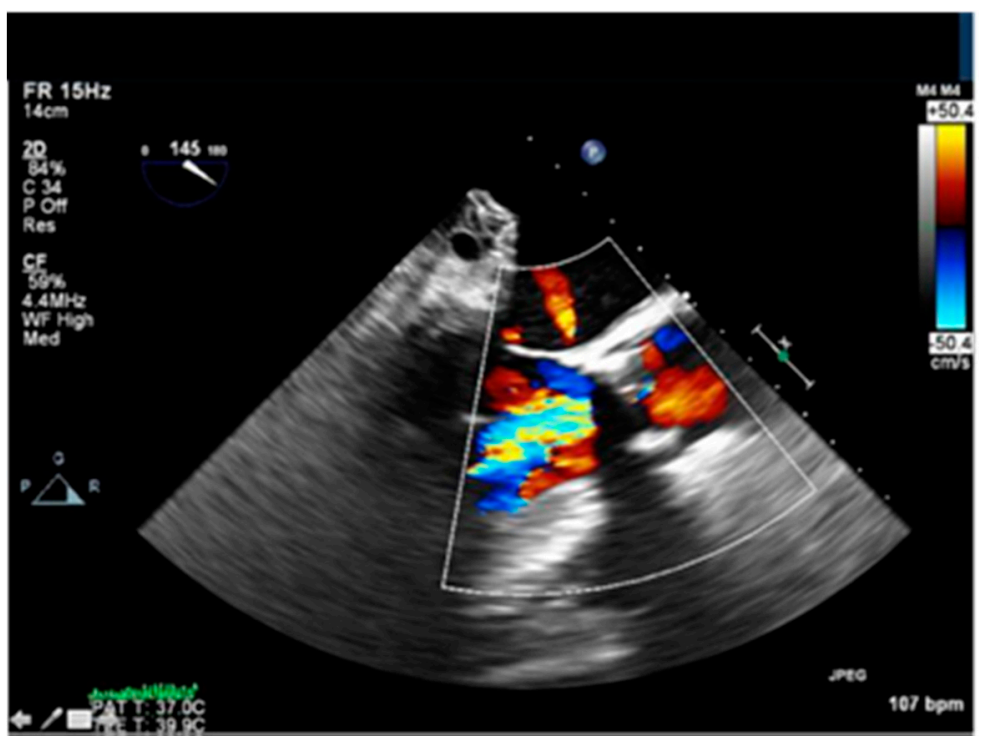

Figure 3. Intraoperative Trans-esophageal echocardiogram showing severe aortic regurgitation. 
Tandem Life ${ }^{\circledR}$ support was increased to $4 \mathrm{~L}$ per minute. At this point, the Gore dry seal sheath was removed from the right femoral artery, and the $26 \mathrm{~mm}$ Medtronic CoreValve EvolutR valve delivery system was introduced via the right femoral artery via its in-line sheath, advanced into position across the valves as shown in Figure 4, and deployed in standard fashion with right ventricular pacing at 100 beats per minute.

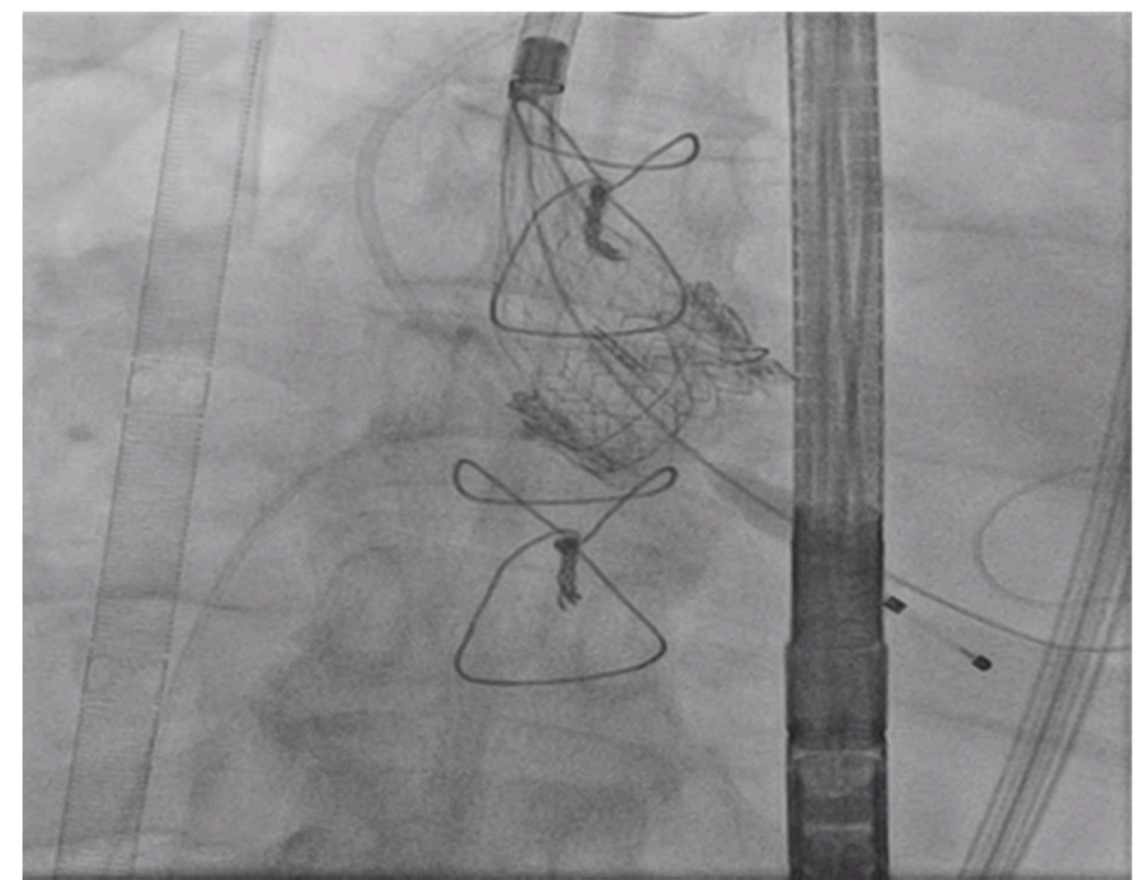

Figure 4. Left Anterior Oblique (LAO) Caudal View: Valve being deployed while transvenous pacemaker in place.

The CoreValve position was confirmed by TEE and fluoroscopy before its deployment. The rotational fluoroscopic view of is shown in Figure 5. TEE and aortogram showed a well-seated transcatheter aortic valve with trace perivalvular leak and good positioning of the prosthesis.

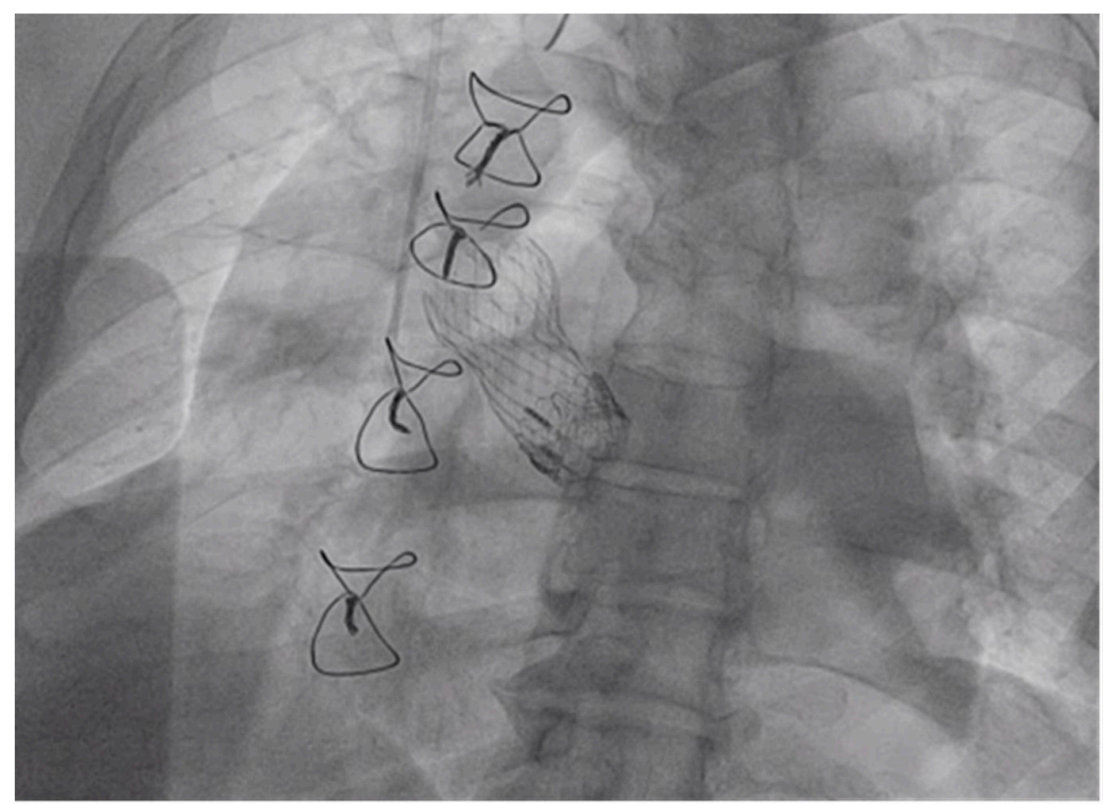

Figure 5. Left Anterior Oblique (LAO) view: Valve is deployed. 
The valve delivery catheter was removed from the wire and the 16 French Gore dry seal sheath was reinserted. A dual lumen pigtail catheter was placed into the left ventricle over the wire and hemodynamic measurements were obtained. The post procedural aortic valve mean gradient was noted to be $11 \mathrm{mmHg}$, and aortic regurgitation was mild with an index of $22 \%$ with trace paravalvular leak as shown in Figure 6.

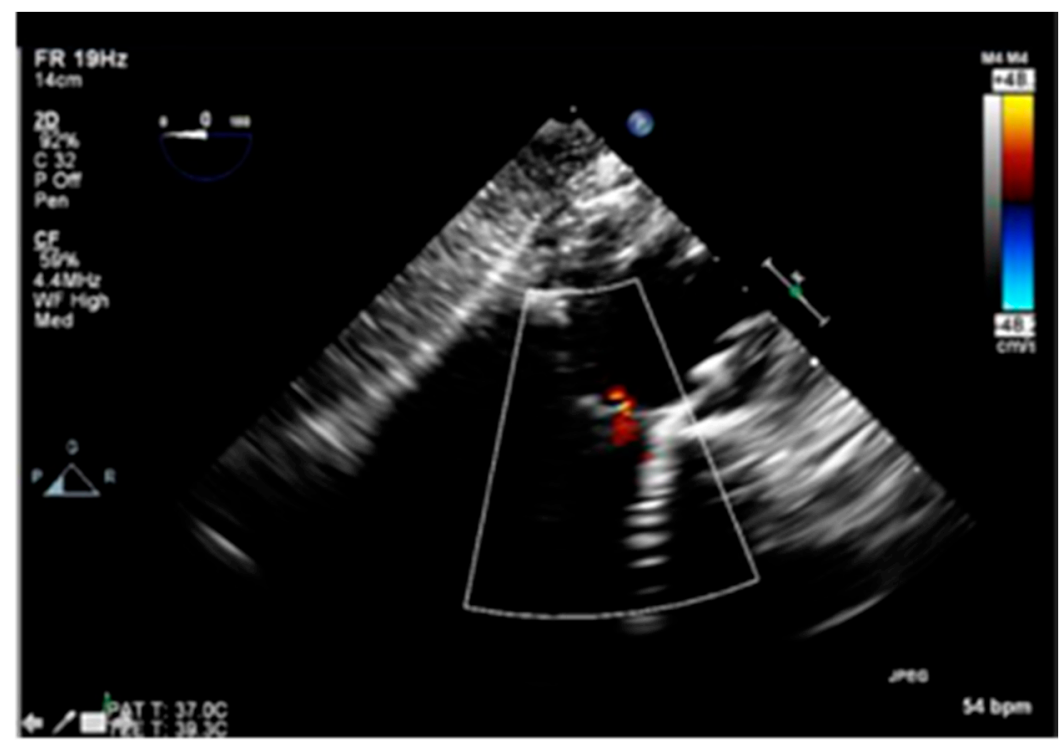

Figure 6. Trans-esophageal echocardiogram after valve deployment showing trace paravalvular leak.

The TandemLife ${ }^{\circledR}$ support was discontinued. The left common artery cannula was removed, and the Perclose sutures were tied. The right common femoral vein cannula and right common femoral artery sheath were removed, and the Perclose sutures were tied. The patient was transferred to the ICU in stable condition and was discharged two days after the procedure. At one month's follow up, TTE showed a well-seated valve with no paravalvular leak and transvalvular mean gradient of $12 \mathrm{mmHg}$.

\section{Discussion}

TAVR is gaining an increasingly important role in the treatment of symptomatic patients with severe AS and is approved by the food and drug administration (FDA) for low, intermediate, high, and extreme risk patients. In recent years, we have seen an increased trend towards the use of bioprostheses due to the increased risk of bleeding and thrombotic complications with mechanical prostheses [1-3]. Despite the benefits, biological tissue used in both surgical and transcatheter bioprostheses is prone to structural valve degeneration (SVD). The incidence of SVD is $\leq 15 \%$ during the first decade post-SAVR, but its incidence progressively increases thereafter [4]. Ten-year follow-up data on TAVR are scarce. Dvir reported a study totaling 378 patients followed for up to 10 years post-TAVR [5]. Out of 35 cases, aortic regurgitation was found in two third of cases, while one third had stenosis or mixed presentation. Clinically relevant SVD would be defined as an increase in mean transvalvular gradient $>20 \mathrm{mmHg}$ with a concomitant decrease in the effective orifice area (EOA) $>0.6 \mathrm{~cm}^{2}$ (and/or decrease in Doppler velocity index $>0.15$ ), leading to severe AS according to current guidelines [6,7].

SVD is a multifactorial process mediated by connective tissue calcification and leaflet degradation leading to valve stenosis and/or regurgitation. Bovine pericardial valves tend to have greater propensity for stenosis whereas porcine valves tend to have greater tendency for leaflet tear. Predictors of surgical aortic bioprosthesis dysfunction include patient-related factors such as lower age and higher BMI, cardiovascular and comorbid factors include smoking, diabetes, dyslipidemia, and renal insufficiency. Valve related factors include left ventricular hypertrophy, small prosthesis size, and prosthesis-patient mismatch. Although long-term data is limited, potential TAVR specific factors that may accelerate structural valve degeneration include leaflet damage during crimping, asymmetric expansion with 
suboptimal leaflet coaptation and incomplete frame expansion leading to leaflet-frame interaction with increased mechanical stress. Combined with an aging population, these factors are likely to result in increasing numbers of patients presenting the structural degeneration of aortic bioprostheses [8].

The treatment for bioprosthetic SVD has conventionally been surgery. However, reoperation for failed surgical valves carries important risks. [9-11] ViV TAVR has recently emerged as a less invasive treatment for patients with degenerated bioprostheses. Along with the factors that affect the feasibility of TAVR in general, the utility of ViV TAVR also is affected by the size and design of the original surgical bioprosthesis. The use of a hemodynamically favorable aortic bioprosthesis at the time of the initial surgical AVR is of special importance, because this can affect the feasibility and outcomes of future ViV TAVR procedures $[9,10]$.

A major concern with ViV TAVR is the development of patient-prosthesis mismatch (PPM). PPM is defined as any situation in which the effective orifice area (EOA) of a prosthetic valve is smaller than the orifice of the patient's native aortic valve; severe aortic PPM is defined as an indexed EOA $\leq 0.65 \mathrm{~cm}^{2} / \mathrm{m}^{2}$ [12]. PPM is especially concerning in ViV TAVR patients as the TAVR prosthesis is implanted within the frame of the previous valve which can result in a smaller EOA. One technique used to prevent the development of PPM is bioprosthetic valve fracture (BVF). This technique is performed by placing a non-compliant valvuloplasty balloon within the surgical prosthetic valve frame and then using high-pressure balloon inflation to fracture the surgical sewing ring of the bioprosthetic valve [13]. Saxon et al. published a review summarizing evidence that supports the concept of fracturing the surgical bioprosthetic valve ring with a high-pressure balloon inflation in order to dilate and permit further expansion of the prosthetic valve area, improving hemodynamic results in such patients [13].

Also discussed is the benefit of using a bioprosthetic aortic valve with supra-annular leaflet positioning (e.g., CoreValve Evolut, Medtronic, Minneapolis, MN, USA) in order to obtain a larger EOA due to the supra-annular position of the prosthetic leaflets, and a higher valve implant depth which may improve inflow dynamics and augment the effective EOA [13-17]. This was the reason we decided to use the Medtronic CoreValve instead of an Edwards SAPIEN valve (Edwards LifeSciences, Irvine, CA, USA) in our patient.

The PARTNER 2 (Placement of Aortic Transcatheter Valves 2) trial was a prospective, multicenter study that enrolled patients with symptomatic AS [4]. ViV procedures were performed in 365 patients and it was concluded that among high-risk patients with degeneration of a bioprosthetic surgical aortic valve, ViV TAVR was associated with better outcomes after one year. The PARTNER 3 trial to assess the safety and effectiveness of the SAPIEN 3 (Edwards Lifesciences, Irvine, CA, USA) in patients with a failing aortic bioprosthetic valve started enrolling patients in January 2017 and the estimated primary completion date is in December 2021.

The VIVID (Valve-In-Valve International Data) Registry is the largest registry to date examining outcomes of the transcatheter valve-in-valve procedure in 459 patients, of whom $30 \%$ had severe prosthetic valve regurgitation and $30 \%$ had combined regurgitation and stenosis. The overall one-year survival rate was $83.2 \%$ [18]. In nonrandomized studies and a systematic review comparing outcomes and safety of the transcatheter valve-in-valve procedure with repeat surgical AVR, the valve-in-valve procedure had similar hemodynamic outcomes, lower stroke risk, and reduced bleeding risk as compared with repeat surgery [19]. The transcather valve in valve procedure has no long-term data available.

Given the above results, among patients at high risk for complications during second surgical AVR, ViV TAVR is an attractive alternative. Current AHA/ACC guidelines state that ViV TAVR is an acceptable alternative second surgery to treat high- and extreme-risk patients with bioprosthetic aortic valve stenosis (stenosis, insufficiency, or combined) in the absence of active infective endocarditis (Class II, LOE A) [6,20]. 


\section{Conclusions}

With the increasing use of TAVR in moderate- and high-risk AS patients and its recent approval in low-risk AS populations, further uses of TAVR within degenerated TAVR valves, as in the case described, should be explored. It is a matter of increased interest given the increasing life expectancy of patients with TAVR and the potential for degeneration of these valves. This case reports repeat ViV TAVR with surgical bioprosthetic valve fracture using a percutaneous cardiopulmonary bypass (TandemLife ${ }^{\circledR}$, Pittsburgh, PA, USA)

Author Contributions: M.A. and S.R. wrote the initial draft, T.K., K.L. and R.S. who were involved in the procedure, proofread it. M.A. worked on the revised version and proofread was done by Rest of team. All authors have read and agree to the published version of the manuscript.

Funding: This research received no external funding.

Conflicts of Interest: The authors declare no conflict of interest.

\section{References}

1. Thourani, V.H.; Suri, R.M.; Gunter, R.L.; Sheng, S.; O’Brien, S.M.; Ailawadi, G.; Szeto, W.Y.; Dewey, T.M.; Guyton, R.A.; Bavaria, J.E.; et al. Contemporary real-world outcomes of surgical aortic valve replacement in 141,905 low-risk, intermediate-risk, and high-risk patients. Ann. Thorac. Surg. 2015, 99, 55-61. [CrossRef] [PubMed]

2. Isaacs, A.J.; Shuhaiber, J.; Salemi, A.; Isom, O.W.; Sedrakyan, A. National trends in utilization and in-hospital outcomes of mechanical versus bioprosthetic aortic valve replacements. J. Thorac. Cardiovasc. Surg. 2015, 149, 1262-1269. [CrossRef] [PubMed]

3. Brennan, J.M.; Edwards, F.H.; Zhao, Y.; O'Brien, S.; Booth, M.E.; Dokholyan, R.S.; Douglas, P.S.; Peterson, E.D.; DEcIDE AVR (Developing Evidence to Inform Decisions about Effectiveness-Aortic Valve Replacement) Research Team. Long-term safety and effectiveness of mechanical versus biologic aortic valve prostheses in older patients: Results from the society of thoracic surgeons adult cardiac surgery national database. Circulation 2013, 127, 1647-1655. [CrossRef] [PubMed]

4. Webb, J.G.; Mack, M.J.; White, J.M.; Dvir, D.; Blanke, P.; Herrmann, H.C.; Leipsic, J.; Kodali, S.K.; Makkar, R.; Miller, D.C.; et al. Transcatheter aortic valve implantation within degenerated aortic surgical bioprostheses, partner 2 valve-in-valve registry. J. Am. Coll. Cardiol. 2017, 69, 2253-2262. [CrossRef] [PubMed]

5. Dvir, D. First look at long-term durability of transcatheter heart valves: Assessment of valve function up to 10 years after implantation. Available online: https://www.crtonline.org/presentation-detail/first-look-atlong-term-durability-of-transcathete (accessed on 1 March 2020).

6. Nishimura, R.A.; Otto, C.M.; Bonow, R.O.; Carabello, B.A.; Erwin, J.P., III; Guyton, R.A.; O'Gara, P.T.; Ruiz, C.E.; Skubas, N.J.; Sorajja, P.; et al. 2014 AHA/ACC guideline for the management of patients with valvular heart disease: A report of the American College of Cardiology/American Heart Association task force on practice guidelines. J. Am. Coll. Cardiol. 2014, 63, e57-e185. [CrossRef] [PubMed]

7. Vahanian, A.; Alfieri, O.; Andreotti, F.; Antunes, M.J.; Barón-Esquivias, G.; Baumgartner, H.; Borger, M.A.; Carrel, T.P.; de Bonis, M.; Evangelista, A.; et al. Guidelines on the management of valvular heart disease (version 2012): The joint task force on the management of valvular heart disease of the european society of cardiology (esc) and the European Association for Cardio-Thoracic Surgery (EACTS). Eur. J. Cardiothorac. Surg. 2012, 42, S1-S44.

8. Rodriguez-Gabella, T.; Voisine, P.; Puri, R.; Pibarot, P.; Rodés-Cabau, J. Aortic bioprosthetic valve durability: Incidence, mechanisms, predictors, and management of surgical and transcatheter valve degeneration. J. Am. Coll. Cardiol. 2017, 70, 1013-1028. [CrossRef] [PubMed]

9. Balsam, L.B.; Grossi, E.A.; Greenhouse, D.G.; Ursomanno, P.; Deanda, A.; Ribakove, G.H.; Culliford, A.T.; Galloway, A.C. Reoperative valve surgery in the elderly: Predictors of risk and long-term survival. Ann. Thorac. Surg. 2010, 90, 1195-1200, discussion 1201. [CrossRef] [PubMed]

10. Kaneko, T.; Loberman, D.; Gosev, I. Reoperative aortic valve replacement in the octogenarians-minimally invasive technique in the era of transcatheter valve replacement. J. Thorac. Cardiovasc. Surg. 2014, 147, 155-162. [CrossRef] [PubMed] 
11. Kaneko, T.; Vassileva, C.M.; Englum, B.; Kim, S.; Yammine, M.; Brennan, M.; Suri, R.M.; Thourani, V.H.; Jacobs, J.P.; Aranki, S. Contemporary outcomes of repeat aortic valve replacement: A benchmark for transcatheter valve-in-valve procedures. Ann. Thorac. Surg. 2015, 100, 1298-1304. [CrossRef] [PubMed]

12. Rahimtoola, S.H. The problem of valve prosthesis-patient mismatch. Circulation 1978, 58, 20-24. [CrossRef] [PubMed]

13. Saxon, J.T. Bioprosthetic valve fracture during valve-in-valve TAVR: Bench to bedside. Interv. Cardiol. Rev. 2018, 13, 20-26. [CrossRef]

14. Allen, K.B.; Chhatriwalla, A.K.; Cohen, D.J.; Chhatriwalla, A.K. Bioprosthetic valve fracture to facilitate transcatheter valve-in-valve implantation. Ann. Thorac. Surg. 2017, 104, 1501-1508. [CrossRef] [PubMed]

15. Chhatriwalla, A.K.; Allen, K.B.; Saxon, J.T.; Cohen, D.J.; Aggarwal, S.; Hart, A.J.; Baron, S.J.; Dvir, D.; Borkon, A.M. Bioprosthetic valve fracture improves the hemodynamic results of valve-in-valve transcatheter aortic valve replacement. Circ. Cardiovasc. Interv. 2017, 10, e005216. [CrossRef] [PubMed]

16. Nielsen-Kudsk, J.E.; Andersen, A.; Therkelsen, C.J.; Christensen, E.H.; Jensen, K.T.; Krusell, L.R.; Tang, M.; Terp, K.A.; Klaaborg, K.; Greisen, J.R.; et al. High-pressure balloon fracturing of small dysfunctional mitroflow bioprostheses facilitates transcatheter aortic valve-in-valve implantation. EuroIntervention 2017, 13, e1020-e1025. [CrossRef] [PubMed]

17. Johansen, P.; Engholt, H.; Tang, M.; Nybo, R.F.; Rasmussen, P.D.; Nielsen-Kudsk, J.E. Fracturing mechanics before valve-in-valve therapy of small aortic bioprosthetic heart valves. EuroIntervention 2017, 13, e1026-e1031. [CrossRef] [PubMed]

18. Dvir, D.; Webb, J.G.; Bleiziffer, S.; Pasic, M.; Waksman, R.; Kodali, S.; Barbanti, M.; Latib, A.; Schaefer, U.; Rodés-Cabau, J. Transcatheter aortic valve implantation in failed bioprosthetic surgical valves. JAMA 2014, 312, 162-170. [CrossRef] [PubMed]

19. Phan, K.; Zhao, D.F.; Wang, N.; Huo, Y.R.; di Eusanio, M.; Yan, T.D. Transcatheter valve-in-valve implantation versus reoperative conventional aortic valve replacement: A systematic review. J. Thorac. Dis. 2016, 8, E83-E93. [PubMed]

20. Nishimura, R.A.; Otto, C.M.; Bonow, R.O.; Carabello, B.A.; Erwin, J.P.; Fleisher, L.A.; Jneid, H.; Mack, M.J.; McLeod, C.J.; O'Gara, P.T.; et al. 2017 AHA/ACC focused update of the 2014 AHA/ACC guideline for the management of patients with valvular heart disease: A report of the American College of Cardiology/American Heart Association task force on clinical practice guidelines. J. Am. Coll. Cardiol. 2017, 70, 252-289. [CrossRef] [PubMed] 